\title{
Intersecting families in a subset of boolean lattices
}

\author{
Jun Wang \\ Department of Mathematics \\ Shanghai Normal University \\ Shanghai 200234, P.R. China \\ jwang@shnu.edu.cn
}

\author{
Huajun Zhang* \\ Department of Mathematics \\ Zhejiang Normal University \\ Jinhua 321004, P.R. China \\ huajunzhang@zjnu.cn
}

Submitted: May 21, 2011; Accepted: Dec 8, 2011; Published: Dec 19, 2011

Mathematics Subject Classifications: 05D05, 06A07

\begin{abstract}
Let $n, r$ and $\ell$ be distinct positive integers with $r<\ell \leq n / 2$, and let $X_{1}$ and $X_{2}$ be two disjoint sets with the same size $n$. Define

$$
\mathcal{F}=\left\{A \in\left(\begin{array}{c}
X \\
r+\ell
\end{array}\right):\left|A \cap X_{1}\right|=r \text { or } \ell\right\},
$$

where $X=X_{1} \cup X_{2}$. In this paper, we prove that if $\mathcal{S}$ is an intersecting family in $\mathcal{F}$, then $|\mathcal{S}| \leq\left(\begin{array}{l}n-1 \\ r-1\end{array}\right)\left(\begin{array}{l}n \\ \ell\end{array}\right)+\left(\begin{array}{l}n-1 \\ \ell-1\end{array}\right)\left(\begin{array}{l}n \\ r\end{array}\right)$, and equality holds if and only if $\mathcal{S}=\{A \in \mathcal{F}: a \in A\}$ for some $a \in X$.
\end{abstract}

Keywords: intersecting family; graded posets; Erdős-Ko-Rado theorem

\section{Introduction}

For a positive integer $n$, let $[n]$ denote the set $\{1,2, \ldots, n\}$, and for a positive integer $k \leq n$, let $[k, n]$ denote the set $\{k, k+1, \ldots, n\}$. Given a set $X$, by $\left(\begin{array}{c}X \\ k\end{array}\right)$ we denote the set of all $k$-subsets of $X$, and by $X \times Y$ we denote the direct product (or Cartesian product) of sets $X$ and $Y$, which consists of all pairs $(x, y)$ where $x \in X$ and $y \in Y$.

A family $\mathcal{A}$ of sets is said to be intersecting if $A \cap B \neq \emptyset$ for every pair $A, B \in \mathcal{A}$. One of the classical results in extremal set theory is the Erdős-Ko-Rado theorem [4]: If $\mathcal{A}$ is

*The corresponding author 
an intersecting family in $\left(\begin{array}{c}{[n]} \\ k\end{array}\right)$, then

$$
|\mathcal{A}| \leq\left(\begin{array}{l}
n-1 \\
k-1
\end{array}\right)
$$

for $n \geq 2 k$, and if $n>2 k$, equality holds if and only if $\mathcal{A}=\left\{A \in\left(\begin{array}{c}{[n]} \\ k\end{array}\right): i \in A\right\}$ for some $i \in X$.

This paper is motivated by the consideration of this theorem from the poset viewpoint. Let $P$ be a finite ranked poset. Thus, $P$ is a poset equipped with a rank function $\rho$ from $P$ into the set of nonnegative integers such that $\rho(x)=0$ for some minimal element $x \in P$, and $\rho(z)=\rho(y)+1$ if $z$ covers $y$ in $P$. The maximum rank of elements of $P$ is denoted by $\rho(P)$. For $0 \leq k \leq \rho(P)$, let $P_{k}$ denote the set of elements with rank $k$. For $x, y \in P$, we say $x$ and $y$ intersect if they have a common lower bound of rank greater than zero. For $P^{\prime} \subseteq P$, let $\alpha\left(P^{\prime}\right)$ denote the maximum size of intersecting families in $P^{\prime}$. And, for $z \in P$ with $\rho(z)>0$, set $P^{\prime}[z]=\left\{x \in P^{\prime}: x \geq z\right\}$. We call $P^{\prime}[z]$ a star (with center $z$ ) if $P^{\prime}[z] \neq \emptyset$. Clearly, a star is an intersecting family in $P$. Hence $\left|P_{k}[z]\right| \leq \alpha\left(P_{k}\right)$. If the equality holds for some $z \in P_{1}$, we then say that $P$ has the EKR property for rank $k$.

In extremal combinatorics, a well-studied poset is the boolean lattice $B_{n}$, consisting of all subsets of $[n]$ ordered by inclusion. It is clear that $B_{n}$ is a ranked poset of rank $n$. Following the above notation, we write its $k$ th rank set as $B_{n, k}$ instead of $\left(\begin{array}{c}{[n]} \\ k\end{array}\right)$. Then, the Erdös-Ko-Rado theorem says that $B_{n}$ has the EKR property for each rank $k \leq n / 2$. It is well known that $B_{n}$ is isomorphic to a direct product of $n$ chains of length one, from which we may find the structures of maximum intersecting families in $B_{n, k}$ for $k<n / 2$. A general definition of direct products of posets is given as follows.

Let $P$ and $Q$ be ranked posets with rank functions $\rho_{P}$ and $\rho_{Q}$, respectively. The direct product of $P$ and $Q$ is a poset defined on $P \times Q$ such that $(x, y) \leq\left(x^{\prime}, y^{\prime}\right)$ if and only if $x \leq x^{\prime}$ in $P$ and $y \leq y^{\prime}$ in $Q$. As usual, this poset is still denoted $P \times Q$. It is easy to see that $P \times Q$ is ranked with the rank function $\rho((x, y))=\rho_{P}(x)+\rho_{Q}(y)$, and

$$
(P \times Q)_{k}=\bigcup_{i+j=k}\left(P_{i} \times Q_{j}\right)
$$

By definition we have that

$$
\alpha\left((P \times Q)_{k}\right) \geq \max \left\{\sum_{i+j=k}\left(\left|P_{i}\left[p_{0}\right]\right| \times\left|Q_{j}\right|\right), \sum_{i+j=k}\left(\left|P_{i}\right| \times\left|Q_{j}\left[q_{0}\right]\right|\right)\right\}
$$

for any $p_{0} \in P_{1}$ and $q_{0} \in Q_{1}$, and equality implies that $P \times Q$ has the EKR property for rank $k$.

Now let us check the boolean lattice. It is well known that $B_{n} \cong B_{m} \times B_{\ell}$ for any positive integers $m$ and $\ell$ with $m+\ell=n$. For any $i_{0} \in[n]$,

$$
\sum_{i+j=k}\left(\left|B_{m, i}\left[i_{0}\right]\right| \times\left|B_{\ell, j}\right|\right)=\sum_{i+j=k}\left(\begin{array}{c}
m-1 \\
i-1
\end{array}\right)\left(\begin{array}{l}
\ell \\
j
\end{array}\right)=\left(\begin{array}{l}
n-1 \\
k-1
\end{array}\right) .
$$


The Erdös-Ko-Rado theorem implies that 1 holds for the direct product $B_{m} \times B_{\ell}$ provided $m+\ell=n \geq 2 k$. This is immediately raise the problem of whether or not the equality holds for other direct products. A related problem is posed by Tardif [13] in the language of graph theory.

Let $P$ be a ranked poset of rank $n$. We say $P$ is rank transitive if there is a group acting transitively on each $P_{i}$ and preserving the order relation of $P$. For every subset $P^{\prime}$ of $P$, we define a graph $G\left[P^{\prime}\right]$, whose vertex set is $P^{\prime}$ and $x y$ is an edge if and only if $x$ and $y$ do not intersecting. Clearly, an intersecting family in $P^{\prime}$ corresponds to an independent set in $G\left[P^{\prime}\right] ; \alpha\left(P_{i}\right)=\alpha\left(G\left[P_{i}\right]\right)$, the independence number of $G\left[P_{i}\right]$, and, if $P$ is rank-transitive, then $G\left[P_{i}\right]$ is vertex-transitive for each $i=0,1, \ldots, n$.

Given graphs $G$ and $H$, the direct product of them is the graph $G \times H$ with vertex set $V(G \times H)=V(G) \times V(H)$ and edge set $E(G \times H)=$ $\left\{\left\{\{u, v\},\left\{u^{\prime}, v^{\prime}\right\}\right\} \quad: \quad\left\{u, u^{\prime}\right\} \in E(G)\right.$ and $\left.\left\{v, v^{\prime}\right\} \in E(H)\right\} . \quad$ Clearly, $\alpha(G \times H) \geq$ $\max \{\alpha(G)|V(H)|, \alpha(H)|V(G)|\}$. In general, the equality does not hold (see [10]). Tardif's problem is whether or not the equality

$$
\alpha(G \times H)=\max \{\alpha(G)|V(H)|, \alpha(H)|V(G)|\}
$$

holds for all vertex-transitive graphs $G$ and $H$. This problem received much attention $[2,5,6,8,9,11,12,15]$. Recently, the second author completely solved this problem [16]. In the language of posets, this result states that if $P$ and $Q$ are ranked and rank-transitive posets, then

$$
\alpha\left(P_{i} \times Q_{j}\right)=\max \left\{\alpha\left(P_{i}\right)\left|Q_{j}\right|,\left|P_{i}\right| \alpha\left(Q_{j}\right)\right\}
$$

hold for all $0 \leq i \leq \rho(P), 0 \leq j \leq \rho(Q)$. Further, we would like to ask, for what kind of ranked posets $P$ and $Q$,

$\alpha\left(\left(P_{i} \times Q_{k-i}\right) \cup\left(P_{j} \times Q_{k-j}\right)\right)=\max \left\{\alpha\left(P_{i}\right)\left|Q_{k-i}\right|+\alpha\left(P_{j}\right)\left|Q_{k-j}\right|,\left|P_{i}\right| \alpha\left(Q_{k-i}\right)+\left|P_{j}\right| \alpha\left(Q_{k-j}\right)\right\}$

hold for all $0 \leq i<j \leq k$. In this paper, we study this problem for boolean lattices.

Let $n, r$ and $\ell$ be distinct positive integers with $r<\ell \leq n / 2$, and let $X_{1}$ and $X_{2}$ be two disjoint sets with the same size $n$. Define

$$
\mathcal{F}=\left\{A \in\left(\begin{array}{c}
X \\
r+\ell
\end{array}\right):\left|A \cap X_{1}\right|=r \text { or } \ell\right\},
$$

where $X=X_{1} \cup X_{2}$. Since $X_{1}$ and $X_{2}$ are disjoint, we may identify $\mathcal{F}$ with a union of two direct products of sets:

$$
\left(\begin{array}{c}
X_{1} \\
r
\end{array}\right) \times\left(\begin{array}{c}
X_{2} \\
\ell
\end{array}\right) \cup\left(\begin{array}{c}
X_{1} \\
\ell
\end{array}\right) \times\left(\begin{array}{c}
X_{2} \\
r
\end{array}\right),
$$

which is clearly isomorphic to $\left(B_{n, r} \times B_{n, \ell}\right) \cup\left(B_{n, \ell} \times B_{n, r}\right)$. If one of $r$ and $\ell$ is greater than $n / 2$, the problem is trivial, and if $r=\ell$, the problem is a special case of Tardif's. So in the following we always assume that $r<\ell \leq n / 2$. The main result in this paper is the following theorem. 
Theorem 1.1 If $\mathcal{S}$ is an intersecting family in $\mathcal{F}$, then

$$
|\mathcal{S}| \leq\left(\begin{array}{l}
n-1 \\
r-1
\end{array}\right)\left(\begin{array}{l}
n \\
\ell
\end{array}\right)+\left(\begin{array}{l}
n-1 \\
\ell-1
\end{array}\right)\left(\begin{array}{l}
n \\
r
\end{array}\right)
$$

and equality holds if and only if $\mathcal{S}=\mathcal{F}[a]=\{A \in \mathcal{F}: a \in A\}$ for some $a \in X$.

Our proof is based on Katona's cycle method [7]. In the next section we expatiate on the way of proving the theorem and present some preliminary results. We then prove the theorem fully in Section 3.

\section{Preliminary Results}

Let $H$ be the graph with vertex set $V(H)=\mathcal{F}$ and edge set $E(H)=\{\{A, B\}$ : $A \cap B=\emptyset$ and $A, B \in \mathcal{F}\}$. Clearly, $H$ is vertex-transitive, and each intersecting subfamily of $\mathcal{F}$ corresponds to an independent set of $H$. So, to prove Theorem 1.1, it suffices to determine the independence number $\alpha(H)$ and the structure of maximum-sized independent sets in $H$.

In the context of vertex-transitive graphs, the following result named "nohomomorphism lemma" is useful to get bounds on the size of independent sets.

Lemma 2.1 (Albertson and Collins [1]) Let $G$ and $G^{\prime}$ be two graphs such that $G$ is vertex-transitive and there exists a homomorphism $\phi: G^{\prime} \mapsto G$. Then $\frac{\alpha(G)}{|V(G)|} \leq \frac{\alpha\left(G^{\prime}\right)}{\left|V\left(G^{\prime}\right)\right|}$, and the equality holds if and only if for any independent set I of cardinality $\alpha(G)$ in $G$, $\phi^{-1}(I)$ is an independent set of cardinality $\alpha\left(G^{\prime}\right)$ in $G^{\prime}$.

This lemma has many applications in extremal combinatorics and graph theory (see $[6,8,9,10,11,12,14,15,16])$. For $B \subset V(G)$, let $G[B]$ denote the sub-graph of $G$ induced by $B$. Then, in Lemma 2.1, by taking $G^{\prime}$ as an induced subgraph $G[B]$ and $\phi$ as the embedding mapping, we obtain the following lemma. For $B \subset V(G)$, let $G[B]$ denote the induced subgraph of $G$ by $B$. Then, in Lemma 2.1, by taking $G^{\prime}$ as an induced subgraph $G[B]$ and $\phi$ as the embedding mapping, we obtain the following lemma (cf. [3]).

Lemma 2.2 (Cameron and $\mathbf{K u )} \frac{\alpha(G)}{|V(G)|} \leq \frac{\alpha(G[B])}{|B|}$ holds for all $B \subseteq V(G)$. Equality implies that $|I \cap B|=\alpha(G[B])$ for every maximum independent set $I$ of $G$.

Clearly, $\mathcal{F}[a]$ is an independent set of $H$ for each $a \in X$, hence $\alpha(H) \geq\left(\begin{array}{l}n-1 \\ r-1\end{array}\right)\left(\begin{array}{l}n \\ \ell\end{array}\right)+$ $\left(\begin{array}{l}n-1 \\ \ell-1\end{array}\right)\left(\begin{array}{l}n \\ r\end{array}\right)$. To prove equality, by Lemma 2.2, we only need to find an induced subgraph $H^{\prime}$ of $H$ with $\frac{\alpha\left(H^{\prime}\right)}{\left|H^{\prime}\right|}=\frac{r+\ell}{2 n}$ so that

$$
\alpha(H) \leq \frac{\alpha\left(H^{\prime}\right)}{\left|H^{\prime}\right|}|H|=\frac{r+\ell}{n}\left(\begin{array}{l}
n \\
r
\end{array}\right)\left(\begin{array}{l}
n \\
\ell
\end{array}\right)=\left(\begin{array}{l}
n-1 \\
r-1
\end{array}\right)\left(\begin{array}{l}
n \\
\ell
\end{array}\right)+\left(\begin{array}{l}
n-1 \\
\ell-1
\end{array}\right)\left(\begin{array}{l}
n \\
r
\end{array}\right) .
$$


We now give some notations. Suppose that $X_{1}=[n]$ and $X_{2}=[n+1,2 n]$. Arrange the elements of $[n]$ on a cycle and let $R_{i}$ and $L_{i}$ denote the $i$ th $r$-interval and $\ell$-interval in the cycle, respectively. That is, for $1 \leq i \leq n, R_{i}$ and $L_{i}$ consist of the least positive residues of $[i, i+r-1]$ and $[i, i+\ell-1]$ modulo $n$, respectively. Similarly, let $R_{i}^{\prime}=\left\{n+x: x \in R_{i}\right\}$ and $L_{i}^{\prime}=\left\{n+y: y \in L_{i}\right\}$. Set $\mathcal{R}=\left\{R_{1}, R_{2}, \ldots, R_{n}\right\}$ and $\mathcal{L}=\left\{L_{1}, L_{2}, \ldots, L_{n}\right\}$, $\mathcal{R}^{\prime}=\left\{R_{1}^{\prime}, R_{2}^{\prime}, \ldots, R_{n}^{\prime}\right\}$ and $\mathcal{L}^{\prime}=\left\{L_{1}^{\prime}, L_{2}^{\prime}, \ldots, L_{n}^{\prime}\right\}$.

Set $\mathcal{H}=\left(\mathcal{R} \times \mathcal{L}^{\prime}\right) \cup\left(\mathcal{L} \times \mathcal{R}^{\prime}\right)$. Then $|\mathcal{H}|=2 n^{2}$ and we may regard $\mathcal{H}$ as a subfamily of $\mathcal{F}$. With the graph $H$ in mind, we consider the induced subgraph $H[\mathcal{H}]$, which contains $H_{0}[\mathcal{R}] \times H_{0}\left[\mathcal{L}^{\prime}\right]$ and $H_{0}[\mathcal{L}] \times H_{0}\left[\mathcal{R}^{\prime}\right]$ as subgraphs, where $H_{0}[\mathcal{R}], H_{0}[\mathcal{L}], H_{0}\left[\mathcal{R}^{\prime}\right]$ and $H_{0}\left[\mathcal{L}^{\prime}\right]$ are defined in a natural way. Clearly, $H_{0}[\mathcal{R}]$ is isomorphic to the well-known circular graph $\operatorname{Circ}(r, n)$. Here, the graph $\operatorname{Circ}(r, n)$ has the vertex set $[n]$, and $i$ and $j$ are not adjacent if and only if $|i-j|<r$ or $|n+i-j|<r$. Hence, $\alpha(\mathcal{R})=n$ if $n<2 r$, and $\alpha(\mathcal{R})=r$ if $n \geq 2 r$. And, when $n>2 r$, by the well-known result of Katona [7], $H_{0}[\mathcal{R}]$ is connected and the maximum-sized independent sets of $H_{0}[\mathcal{R}]$ are stars.

We shall prove that $\alpha(H[\mathcal{H}])=(r+\ell) n$, implying $\frac{\alpha(H[\mathcal{H}])}{|H[\mathcal{H}]|}=\frac{r+\ell}{2 n}$, i.e, the induced subgraph $H[\mathcal{H}]$ is a desired subgraph $H^{\prime}$. To do this, we first present a lemma.

For $\mathcal{C}, \mathcal{D} \subseteq \mathcal{R} \cup \mathcal{R}^{\prime} \cup \mathcal{L} \cup \mathcal{L}^{\prime}$, set $N_{\mathcal{D}}(\mathcal{C})=\{A \in \mathcal{D}: A \cap B=\emptyset$ for some $B \in \mathcal{C}\}$ and $\bar{N}_{\mathcal{D}}(\mathcal{C})=\mathcal{D} \backslash N_{\mathcal{D}}(\mathcal{C})=\{A \in \mathcal{D}: A \cap B \neq \emptyset$ for all $B \in \mathcal{C}\}$.

Lemma 2.3 Let $\mathcal{C}, \mathcal{D} \in\{\mathcal{R}, \mathcal{L}\}$. For each $\mathcal{A} \subseteq \mathcal{C}$, we have that $\bar{N}_{\mathcal{D}}(\mathcal{A})=\emptyset$ if $|\mathcal{A}| \geq$ $\alpha(\mathcal{C})+\alpha(\mathcal{D})$; and $\left|\bar{N}_{\mathcal{D}}(\mathcal{A})\right|+|\mathcal{A}| \leq \alpha(\mathcal{C})+\alpha(\mathcal{D})$ if $|\mathcal{A}|<\alpha(\mathcal{C})+\alpha(\mathcal{D})$. If $\left|\bar{N}_{\mathcal{D}}(\mathcal{A})\right|+$ $|\mathcal{A}|=\alpha(\mathcal{C})+\alpha(\mathcal{D})$, then $\mathcal{A}=\left\{R_{i}, R_{i+1}, \ldots, R_{i+|\mathcal{A}|-1}\right\}$ or $\left\{L_{i}, L_{i+1}, \ldots, L_{i+|\mathcal{A}|-1}\right\}$ for some $i \in[n]$, according to $\mathcal{C}=\mathcal{R}$ or $\mathcal{L}$.

Proof. Suppose $\mathcal{C}=\mathcal{R}$ and $\mathcal{D}=\mathcal{L}$. Then $\alpha(\mathcal{C})=r$ and $\alpha(\mathcal{D})=\ell$. For $L_{i} \in \mathcal{L}$, it is clear that $\mathcal{A} \subseteq \bar{N}_{\mathcal{R}}\left(\left\{L_{i}\right\}\right)$ if and only if $L_{i} \in \bar{N}_{\mathcal{L}}(\mathcal{A})$. By definition it is easy to count that $\left|\bar{N}_{\mathcal{R}}\left(\left\{L_{i}\right\}\right)\right|=r+\ell-1$ for every $L_{i} \in \mathcal{L}$. Therefore, if $|\mathcal{A}| \geq r+\ell$, then $\mathcal{A}$ cannot be a subset of $\bar{N}_{\mathcal{R}}\left(\left\{L_{i}\right\}\right)$, i.e., no $L_{i}$ 's belong to $\bar{N}_{\mathcal{L}}(\mathcal{A})$. This proves that $\bar{N}_{\mathcal{L}}(\mathcal{A})=\emptyset$ if $|\mathcal{A}| \geq r+\ell$. Suppose that $|\mathcal{A}|=s \leq r+\ell-1$ and $\bar{N}_{\mathcal{L}}(\mathcal{A}) \neq \emptyset$. By symmetry we may assume that $L_{r} \in \bar{N}_{\mathcal{L}}(\mathcal{A})$. Then $\mathcal{A} \subseteq \bar{N}_{\mathcal{R}}\left(\left\{L_{r}\right\}\right)=\left\{R_{1}, R_{2}, \ldots, R_{r+\ell-1}\right\}$. So we may assume that $\mathcal{A}=\left\{R_{i_{1}}, R_{i_{2}}, \ldots, R_{i_{s}}\right\}$ with $1 \leq i_{1}<i_{2}<\cdots<i_{s} \leq r+\ell-1$. Set $\mathcal{A}_{j}=\left\{R_{i_{1}}, R_{i_{2}}, \ldots, R_{i_{j}}\right\}$ for $1 \leq j \leq s$. Then $\bar{N}_{\mathcal{L}}\left(\mathcal{A}_{1}\right) \supseteq \bar{N}_{\mathcal{L}}\left(\mathcal{A}_{2}\right) \supseteq \cdots \supseteq \bar{N}_{\mathcal{L}}\left(\mathcal{A}_{s}\right)=$ $\bar{N}_{\mathcal{L}}(\mathcal{A})$. Note that $\bar{N}_{\mathcal{L}}\left(\left\{R_{i}\right\}\right)=\left\{L_{i+1-\ell}, L_{i+2-\ell}, \ldots, L_{i+r-1}\right\}$ for each $R_{i} \in \mathcal{R}$. It is clear that $L_{i_{j+1}-\ell} \notin \bar{N}_{\mathcal{L}}\left(\mathcal{A}_{j+1}\right)$. On the other hand, because $-\ell<i_{t}-\left(i_{j+1}-r\right)=r-i_{j+1}+$ $i_{t}<r$ for $t=1,2, \ldots, j$, we have that $L_{i_{j+1}-r} \in \bar{N}_{\mathcal{L}}\left(\left\{R_{i_{t}}\right\}\right)$ for $1 \leq t \leq j$. Therefore $r+\ell-1=\left|\bar{N}_{\mathcal{L}}\left(\mathcal{A}_{1}\right)\right|>\left|\bar{N}_{\mathcal{L}}\left(\mathcal{A}_{2}\right)\right|>\cdots>\left|\bar{N}_{\mathcal{L}}\left(\mathcal{A}_{s-1}\right)\right|>\left|\bar{N}_{\mathcal{L}}(\mathcal{A})\right|$, which implies that $\left|\bar{N}_{\mathcal{L}}(\mathcal{A})\right|+|\mathcal{A}| \leq r+\ell$. Furthermore, since $n>r+\ell$, if $i_{j+1}>i_{j}+1$, it is easy to show that $L_{i_{j}+1-\ell} \notin \bar{N}_{\mathcal{L}}\left(\mathcal{A}_{j+1}\right)$ but $L_{i_{j}+1-\ell} \in \bar{N}_{\mathcal{L}}\left(\mathcal{A}_{j}\right)$, that is, $\left|\bar{N}_{\mathcal{L}}\left(\mathcal{A}_{j}\right)\right|>\left|\bar{N}_{\mathcal{L}}\left(\mathcal{A}_{j+1}\right)\right|+1$. Therefore, $\left|\bar{N}_{\mathcal{L}}(\mathcal{A})\right|+|\mathcal{A}|=r+\ell$ holds if and only if $\mathcal{A}=\left\{R_{i}, R_{i+1}, \ldots, R_{i+|\mathcal{A}|-1}\right\}$ for some $i \in[n]$.

The other cases can be settled in a similar way, so we omit the detail. 


\section{Proof of Theorem 1.1}

Let $\mathcal{F}$ and $\mathcal{H}$ be defined as above and let $\mathcal{S}$ and $\mathcal{S}^{\prime}$ be maximum-sized intersecting families in $\mathcal{F}$ and $\mathcal{H}$, respectively. The proof of the theorem is completed in two steps: (i) $\left|\mathcal{S}^{\prime}\right|=n(r+\ell)$, and (ii) $\mathcal{S}$ is a star.

We first prove (i). For $i \in[n]$, let us consider the star $\mathcal{H}[i]=\{A \in \mathcal{H}: i \in A\}$. Then, the maximality of $\left|\mathcal{S}^{\prime}\right|$ implies that $\left|\mathcal{S}^{\prime}\right| \geq|\mathcal{H}[i]|=n(r+\ell)$. We now proceed to prove that $\mathcal{S}^{\prime}=\mathcal{H}[i]$ for some $i \in[n]$, which would complete the first step of the proof.

Given $A \in \mathcal{R} \cup \mathcal{L}$, define

$$
\mathcal{S}_{A}^{\prime}=\left\{C \in \mathcal{R}^{\prime} \cup \mathcal{L}^{\prime}:(A, C) \in \mathcal{S}^{\prime}\right\}
$$

and

$$
\mathcal{P}_{A}= \begin{cases}\mathcal{L}^{\prime}, & \text { if } A \in \mathcal{R} ; \\ \mathcal{R}^{\prime}, & \text { if } A \in \mathcal{L} .\end{cases}
$$

Clearly, $\mathcal{S}_{A}^{\prime} \subseteq \mathcal{P}_{A}$ for $A \in \mathcal{R} \cup \mathcal{L}$. Set

$$
\begin{array}{ll}
\mathcal{R}_{1}=\left\{A \in \mathcal{R}:\left|\mathcal{S}_{A}^{\prime}\right|>\ell\right\}, & \mathcal{L}_{1}=\left\{B \in \mathcal{L}:\left|\mathcal{S}_{B}^{\prime}\right|>r\right\}, \\
\mathcal{R}_{2}=\left\{A \in \mathcal{R}: 0<\left|\mathcal{S}_{A}^{\prime}\right| \leq \ell\right\}, & \mathcal{L}_{2}=\left\{B \in \mathcal{L}: 0<\left|\mathcal{S}_{B}^{\prime}\right| \leq r\right\}
\end{array}
$$

and set $\left.\mathcal{S}^{\prime}\right|_{X_{1}}=\mathcal{R}_{1} \cup \mathcal{R}_{2} \cup \mathcal{L}_{1} \cup \mathcal{L}_{2}$. That is, $\left.\mathcal{S}^{\prime}\right|_{X_{1}}$ is the projection of $\mathcal{S}^{\prime}$ on $\left(\begin{array}{c}X_{1} \\ r\end{array}\right) \cup\left(\begin{array}{c}X_{1} \\ \ell\end{array}\right)$. From this observation it follows that

$$
\left|\mathcal{S}^{\prime}\right|=\sum_{\left.A \in \mathcal{S}^{\prime}\right|_{X_{1}}}\left|S_{A}^{\prime}\right| .
$$

For any $A,\left.B \in \mathcal{S}^{\prime}\right|_{X_{1}}$, if $A \cap B=\emptyset$, then $C \cap D \neq \emptyset$ holds for all $C \in \mathcal{S}_{A}^{\prime}$ and $D \in \mathcal{S}_{B}^{\prime}$ because $\mathcal{S}^{\prime}$ is an intersecting family. Then $\mathcal{S}_{B}^{\prime} \subseteq \bar{N}_{\mathcal{P}_{B}}\left(\mathcal{S}_{A}^{\prime}\right)$, which implies that $\left|\mathcal{S}_{A}^{\prime}\right|+\left|\bar{N}_{\mathcal{P}_{B}}\left(\mathcal{S}_{A}^{\prime}\right)\right| \geq\left|\mathcal{S}_{A}^{\prime}\right|+\left|\mathcal{S}_{B}^{\prime}\right|$. By Lemma 2.3, however, we have $\left|\mathcal{S}_{A}^{\prime}\right|+\left|\bar{N}_{\mathcal{P}_{B}}\left(\mathcal{S}_{A}^{\prime}\right)\right| \leq$ $\alpha\left(\mathcal{P}_{A}\right)+\alpha\left(\mathcal{P}_{B}\right)$. Therefore, we have the following claim.

Claim: For any $A,\left.B \in \mathcal{S}^{\prime}\right|_{X_{1}}$, if $A \cap B=\emptyset$, then $\left|\mathcal{S}_{A}^{\prime}\right|+\left|\mathcal{S}_{B}^{\prime}\right| \leq \alpha\left(\mathcal{P}_{A}\right)+\alpha\left(\mathcal{P}_{B}\right)$.

By the claim we immediately obtain that $A \cap B \neq \emptyset$ for any $A, B \in \mathcal{R}_{1} \cup \mathcal{L}_{1}$. Therefore, $\mathcal{R}_{1} \cup \mathcal{L}_{1}$ is an intersecting family in $\mathcal{R} \cup \mathcal{L}$.

Set $\mathcal{D}_{1}=\left\{A \in \mathcal{R}_{1} \cup \mathcal{L}_{1}: N_{\mathcal{D}_{2}}(\{A\})=\emptyset\right\}, \mathcal{D}_{1}^{\prime}=\left\{A \in \mathcal{R}_{1} \cup \mathcal{L}_{1}: N_{\mathcal{D}_{2}}(\{A\}) \neq \emptyset\right\}$, where $\mathcal{D}_{2}=\mathcal{R}_{2} \cup \mathcal{L}_{2}$. By definition we have immediately that $\left\{\mathcal{D}_{1}, \mathcal{D}_{1}^{\prime}, \mathcal{D}_{2}\right\}$ is a partition of $\left.\mathcal{S}^{\prime}\right|_{X_{1}}, \mathcal{D}_{1} \cup \mathcal{D}_{1}^{\prime}=\mathcal{R}_{1} \cup \mathcal{L}_{1}$, and $\mathcal{D}_{1}$ and $\mathcal{D}_{1}^{\prime} \cup \mathcal{D}_{2}$ are cross-intersecting, i.e., $A \cap B \neq \emptyset$ for all $A \in \mathcal{D}_{1}$ and $B \in \mathcal{D}_{1}^{\prime} \cup \mathcal{D}_{2}$.

If $\mathcal{D}_{2}=\emptyset$, then $\mathcal{S}^{\prime} \subseteq\left(\mathcal{R}_{1} \times \mathcal{L}^{\prime}\right) \cup\left(\mathcal{L}_{1} \times \mathcal{R}^{\prime}\right)$. So $\left|\mathcal{S}^{\prime}\right| \leq n\left(\left|\mathcal{R}_{1}\right|+\left|\mathcal{L}_{1}\right|\right) \leq n(\alpha(\mathcal{R})+\alpha(\mathcal{L}))=$ $n(r+\ell)$, and the equality holds if and only if both $\mathcal{R}_{1}$ and $\mathcal{L}_{1}$ are stars of order $r$ and $\ell$, respectively. Therefore, the maximality of $\mathcal{S}^{\prime}$ implies that $\mathcal{S}^{\prime}=\mathcal{H}[i]$ for some $i \in X_{1}$. So in the following we suppose $\mathcal{D}_{2} \neq \emptyset$, and prove that $\mathcal{D}_{1} \cup \mathcal{D}_{1}^{\prime}=\emptyset$.

We first prove that $\mathcal{D}_{1}^{\prime}=\emptyset$. Suppose contrary that $\mathcal{D}_{1}^{\prime} \neq \emptyset$. Then $\left|N_{\mathcal{D}_{2}}(\{A\})\right| \geq 1$ for every $A \in \mathcal{D}_{1}^{\prime}$. Assume that $t \geq 1$ and $\left|N_{\mathcal{D}_{2}}(\mathcal{D})\right| \geq|\mathcal{D}|$ holds for all $\mathcal{D} \subseteq \mathcal{D}_{1}^{\prime}$ whenever $|\mathcal{D}| \leq t$. We now prove that, if $t<\left|\mathcal{D}_{1}^{\prime}\right|$, then every $(t+1)$-subset of $\mathcal{D}_{1}^{\prime}$ also has 
this property. Otherwise, there is a $(t+1)$-subset of $\mathcal{D}_{1}^{\prime}$, say $\mathcal{D}^{\prime}=\mathcal{D} \cup\left\{A^{\prime}\right\}$, satisfying $\left|N_{\mathcal{D}_{2}}\left(\mathcal{D}^{\prime}\right)\right|=t=\left|N_{\mathcal{D}_{2}}(\mathcal{D})\right|$. Set

$$
\mathcal{S}_{1}^{\prime}=\left[\mathcal{S}^{\prime}-\cup_{A \in N_{\mathcal{D}_{2}}(\mathcal{D})}\left(\{A\} \times \mathcal{S}_{A}^{\prime}\right)\right] \bigcup\left[\cup_{A \in \mathcal{D}^{\prime}}\left(\{A\} \times \mathcal{P}_{A}\right)\right]
$$

It is not difficult to see that $\mathcal{S}_{1}^{\prime}$ is also an intersecting family in $\mathcal{H}$ because $\mathcal{D}_{1} \cup \mathcal{D}_{1}^{\prime}$ is an intersecting family so that $N_{\left.\mathcal{S}^{\prime}\right|_{X_{1}}}\left(\mathcal{D}^{\prime}\right)=N_{\mathcal{D}_{2}}\left(\mathcal{D}^{\prime}\right)$. Set $\mathcal{D}=\left\{A_{1}, A_{2}, \ldots, A_{t}\right\}$. By Hall's marriage Theorem, we may rearrange the elements of $N_{\mathcal{D}_{2}}(\mathcal{D})$ so that $N_{\mathcal{D}_{2}}(\mathcal{D})=$ $\left\{B_{1}, B_{2}, \ldots, B_{t}\right\}$ with $A_{i} \cap B_{i}=\emptyset$ for $i=1,2, \ldots, t$. Note that $\left|\mathcal{S}_{A^{\prime}}^{\prime}\right|<n$. Then, by the claim, we can deduce that

$$
\left|\mathcal{S}_{1}^{\prime}\right|-\left|\mathcal{S}^{\prime}\right|=\left(n-\left|\mathcal{S}_{A^{\prime}}^{\prime}\right|\right)+\sum_{1 \leq i \leq t}\left(n-\left|\mathcal{S}_{A_{i}}^{\prime}\right|-\left|\mathcal{S}_{B_{i}}^{\prime}\right|\right)>0
$$

contradicting the maximality of $\left|\mathcal{S}^{\prime}\right|$. We therefore obtain that $\left|N_{\mathcal{D}_{2}}\left(\mathcal{D}_{1}^{\prime}\right)\right| \geq\left|\mathcal{D}_{1}^{\prime}\right|$. Set $\mathcal{D}_{1}^{\prime}=\left\{A_{1}, A_{2}, \ldots, A_{s}\right\}$ and assume $B_{1}, B_{2}, \ldots, B_{s} \in N_{\mathcal{D}_{2}}\left(\mathcal{D}_{1}^{\prime}\right)$ such that $A_{i} \cap B_{i}=\emptyset$, $i=1,2, \ldots, s$. If $\left|N_{\mathcal{D}_{2}}\left(\mathcal{D}_{1}^{\prime}\right)\right|>s$, set

$$
\mathcal{S}_{2}^{\prime}=\left[\cup_{A \in \mathcal{D}_{1}}\left(\{A\} \times \mathcal{P}_{A}\right)\right] \cup\left[\cup_{A \in \mathcal{D}_{1}^{\prime} \cup \mathcal{D}_{2}}\left(\{A\} \times \mathcal{P}_{A}[a]\right)\right]
$$

where $a \in X_{2}$. Then $\mathcal{S}_{2}^{\prime}$ is an intersecting family because both $\cup_{A \in \mathcal{D}_{1}}\left(\{A\} \times \mathcal{P}_{A}\right)$ and $\cup_{A \in \mathcal{D}_{1}^{\prime} \cup \mathcal{D}_{2}}\left(\{A\} \times \mathcal{P}_{A}[a]\right)$ are intersecting families, and $\mathcal{D}_{1}$ and $\mathcal{D}_{1}^{\prime} \cup \mathcal{D}_{2}$ are cross-intersecting. And,

$$
\begin{aligned}
\left|\mathcal{S}_{2}^{\prime}\right|-\left|\mathcal{S}^{\prime}\right|= & \sum_{A \in \mathcal{D}_{1}}\left(n-\left|\mathcal{S}_{A}^{\prime}\right|\right)+\sum_{A \in \mathcal{D}_{1}^{\prime} \cup \mathcal{D}_{2}}\left(\alpha\left(\mathcal{P}_{A}\right)-\left|\mathcal{S}_{A}^{\prime}\right|\right) \\
= & \sum_{A \in \mathcal{D}_{1}}\left(n-\left|\mathcal{S}_{A}^{\prime}\right|\right)+\sum_{1 \leq i \leq s}\left(\alpha\left(\mathcal{P}_{A_{i}}\right)+\alpha\left(\mathcal{P}_{B_{i}}\right)-\left|\mathcal{S}_{A_{i}}^{\prime}\right|-\left|\mathcal{S}_{B_{i}}^{\prime}\right|\right) \\
& +\sum_{B \in \mathcal{D}_{2}^{\prime}}\left(\alpha\left(\mathcal{P}_{B}\right)-\left|\mathcal{S}_{B}^{\prime}\right|\right),
\end{aligned}
$$

where $\mathcal{D}_{2}^{\prime}=\mathcal{D}_{2}-\left\{B_{1}, B_{2} \ldots, B_{s}\right\}$. Clearly, $n \geq\left|\mathcal{S}_{A}^{\prime}\right|$ for any $A \in \mathcal{D}_{1}$, and by the claim, $\alpha\left(\mathcal{P}_{A_{i}}\right)+\alpha\left(\mathcal{P}_{B_{i}}\right) \geq\left|\mathcal{S}_{A_{i}}^{\prime}\right|+\left|\mathcal{S}_{B_{i}}^{\prime}\right|$ for $i=1,2, \ldots, s$. By definition, $\alpha\left(\mathcal{P}_{B}\right) \geq\left|\mathcal{S}_{B}^{\prime}\right|$ for all $B \in \mathcal{D}_{2}^{\prime}$, and, because $\left|N_{\mathcal{D}_{2}}\left(\mathcal{D}_{1}^{\prime}\right)\right|>\left|\mathcal{D}_{1}^{\prime}\right|$, there exists a $B^{\prime} \in \mathcal{D}_{2}^{\prime}$ and $A_{j} \in \mathcal{D}_{1}^{\prime}$ with $B^{\prime} \cap A_{j}=\emptyset$. Then, the claim implies $\alpha\left(\mathcal{P}_{B^{\prime}}\right)+\alpha\left(\mathcal{P}_{A_{j}}\right) \geq\left|\mathcal{S}_{B^{\prime}}^{\prime}\right|+\left|\mathcal{S}_{A_{j}}^{\prime}\right|$. By definition, however, $\left|\mathcal{S}_{A_{j}}^{\prime}\right|>\alpha\left(\mathcal{P}_{A_{j}}\right)$ for $A_{j} \in \mathcal{D}_{1}$. Hence $\alpha\left(\mathcal{P}_{B^{\prime}}\right)>\left|\mathcal{S}_{B^{\prime}}^{\prime}\right|$. We thus proved that $\left|\mathcal{S}_{2}^{\prime}\right|>\left|\mathcal{S}^{\prime}\right|$, contradicting the maximality of $\left|\mathcal{S}^{\prime}\right|$. Therefore, $\left|\mathcal{D}_{1}^{\prime}\right|=\left|N_{\mathcal{D}_{2}}\left(\mathcal{D}_{1}^{\prime}\right)\right|$.

In order to show $\mathcal{D}_{1}^{\prime}=\emptyset$, we construct another family as follows:

$$
\mathcal{S}_{3}^{\prime}=\left[\mathcal{S}^{\prime}-\cup_{1 \leq i \leq s}\left(\left\{B_{i}\right\} \times \mathcal{S}_{B_{i}}^{\prime}\right)\right] \cup\left[\cup_{1 \leq i \leq s}\left(\left\{A_{i}\right\} \times \mathcal{P}_{A}\right)\right]
$$

Clearly, $\mathcal{S}_{3}^{\prime}$ is an intersecting family in $\mathcal{H}$. Using the similar argument to that for $\mathcal{S}_{2}^{\prime}$ we have that

$$
\begin{aligned}
\left|\mathcal{S}_{3}^{\prime}\right|-\left|\mathcal{S}^{\prime}\right| & =\sum_{1 \leq i \leq s}\left(n-\left|\mathcal{S}_{A_{i}}^{\prime}\right|-\left|\mathcal{S}_{B_{i}}^{\prime}\right|\right) \geq \sum_{1 \leq i \leq s}\left(n-\alpha\left(\mathcal{P}_{A_{i}}\right)-\alpha\left(\mathcal{P}_{B_{i}}\right)\right) \\
& \geq s(n-2 \ell) \geq 0 .
\end{aligned}
$$


From this we see that $\left|\mathcal{S}_{3}^{\prime}\right|=\left|\mathcal{S}^{\prime}\right|$ if and only if $\left|\mathcal{S}_{A_{i}}^{\prime}\right|=\left|\mathcal{S}_{B_{i}}^{\prime}\right|=\alpha\left(\mathcal{P}_{A_{i}}\right)=\alpha\left(\mathcal{P}_{B_{i}}\right)=$ $\ell=\frac{n}{2}$. Suppose it is the case. Then $\mathcal{D}_{1}^{\prime} \cup N_{\mathcal{D}_{2}}\left(\mathcal{D}_{1}^{\prime}\right) \subset \mathcal{R}$, and then $\mathcal{L}_{1} \subset \mathcal{D}_{1}$. Set $\mathcal{R}_{2}^{\prime}=\mathcal{R}_{2}-N_{\mathcal{D}_{2}}\left(\mathcal{D}_{1}^{\prime}\right)$. If $\mathcal{L}_{1} \neq \emptyset$, then by definition of $\mathcal{R}_{2}^{\prime}$ and $\mathcal{R}_{1} \cup \mathcal{L}_{1}$ is intersecting, $\mathcal{R}_{1} \cup \mathcal{R}_{2}^{\prime} \subseteq \bar{N}_{\mathcal{R}}\left(\mathcal{L}_{1}\right)$, and then by Lemma 2.3 ,

$$
\left|\mathcal{R}_{1}\right|+\left|\mathcal{R}_{2}^{\prime}\right|+\left|\mathcal{L}_{1}\right| \leq r+\ell .
$$

Clearly, if $\mathcal{L}_{1}=\emptyset$ and $\mathcal{R}_{2}^{\prime}=\emptyset$, then $\left|\mathcal{R}_{1}\right|+\left|\mathcal{R}_{2}^{\prime}\right|+\left|\mathcal{L}_{1}\right| \leq r<r+\ell$, because $\mathcal{R}_{1}$ is an intersecting family in $\mathcal{R}$. If $\mathcal{L}_{1}=\emptyset$ but $\mathcal{R}_{2}^{\prime} \neq \emptyset$, then since $\mathcal{R}_{2}^{\prime} \subseteq \bar{N}_{\mathcal{R}}\left(\mathcal{R}_{1}\right)$, Lemma 2.3 implies $\left|\mathcal{R}_{1}\right|+\left|\mathcal{R}_{2}^{\prime}\right| \leq 2 r<r+\ell$. Therefore, (4) holds in any cases. Similarly, we can also obtain that the inequality

$$
\left|\mathcal{R}_{1}\right|+\left|\mathcal{L}_{1}\right|+\left|\mathcal{L}_{2}\right| \leq r+\ell
$$

always holds.

Suppose $\mathcal{L}_{2}=\emptyset$. Then $\mathcal{S}_{3}^{\prime} \subseteq\left(\mathcal{R} \times \mathcal{L}^{\prime}\right) \cup\left(\mathcal{L}_{1} \times \mathcal{R}^{\prime}\right)$. Recall that $\left|\mathcal{S}_{3}^{\prime}\right| \geq\left|\mathcal{S}^{\prime}\right| \geq n(r+\ell)$. If we assume that $\mathcal{L}_{1}=\emptyset$, then we get $\left|\mathcal{S}_{3}^{\prime}\right| \leq\left|\mathcal{R}_{1}\right| n+\left|\mathcal{R}_{2}\right| \ell \leq\left|\mathcal{R}_{1}\right| n+\left(n-\left|\mathcal{R}_{1}\right|\right) \ell=$ $n \ell+\left|\mathcal{R}_{1}\right|(n-\ell) \leq n \ell+r(n-\ell) \leq n(r+\ell)$, a contradiction. So $\mathcal{L}_{1} \neq \emptyset$. Since we earlier obtained $\mathcal{L}_{1} \subseteq \mathcal{D}_{1}$, we have $\mathcal{R}_{2} \subseteq \bar{N}_{\mathcal{R}}\left(\mathcal{L}_{1}\right)$, and together with the fact that $\mathcal{R}_{1} \cup \mathcal{L}_{1}$ is intersecting, this implies that $\mathcal{R}_{1} \cup \mathcal{R}_{2} \subseteq \bar{N}_{\mathcal{R}}\left(\mathcal{L}_{1}\right)$. Then, Lemma 2.3 implies that $\left|\mathcal{R}_{1}\right|+\left|\mathcal{R}_{2}\right|+\left|\mathcal{L}_{1}\right| \leq r+\ell$. By the definition of $\mathcal{R}_{2}$ and the above properties of $\mathcal{S}_{3}^{\prime}$, it follows that $\left|\mathcal{R}_{2}\right|=0$. But then, since $\mathcal{D}_{2}=\mathcal{R}_{2} \cup \mathcal{L}_{2}$, we get $\mathcal{D}_{2}=\emptyset$, a contradiction. Therefore, $\mathcal{L}_{2} \neq \emptyset$. Recall that $\ell=\frac{n}{2}>r$. From (3) it follows that

$$
\begin{aligned}
\left|\mathcal{S}_{3}^{\prime}\right| & \leq n\left(\left|\mathcal{R}_{1}\right|+\left|\mathcal{L}_{1}\right|\right)+\ell\left|\mathcal{R}_{2}^{\prime}\right|+r\left|\mathcal{L}_{2}\right| \\
& =n\left(\left|\mathcal{R}_{1}\right|+\left|\mathcal{R}_{2}^{\prime}\right|+\left|\mathcal{L}_{1}\right|\right)-\ell\left|\mathcal{R}_{2}^{\prime}\right|+r\left|\mathcal{L}_{2}\right| \\
& =n\left(\left|\mathcal{R}_{1}\right|+\left|\mathcal{L}_{1}\right|+\left|\mathcal{L}_{2}\right|\right)+\ell\left|\mathcal{R}_{2}^{\prime}\right|+(r-n)\left|\mathcal{L}_{2}\right|,
\end{aligned}
$$

from which, together with (4) and (5), it follows that $\left|\mathcal{S}_{3}^{\prime}\right|<n(r+\ell)$, yielding a contradiction. Therefore, $\mathcal{D}_{1}^{\prime}=\emptyset$.

If $\mathcal{D}_{1} \neq \emptyset$, then $\left|\mathcal{S}^{\prime}\right| \leq n\left|\mathcal{D}_{1}\right|+\ell\left|\mathcal{R}_{2}\right|+r\left|\mathcal{L}_{2}\right|=n\left(\left|\mathcal{R}_{1}\right|+\left|\mathcal{L}_{1}\right|\right)+\ell\left|\mathcal{R}_{2}\right|+r\left|\mathcal{L}_{2}\right|$. Similarly to (4) and (5), we can also obtain that the two inequalities

$$
\left|\mathcal{R}_{1}\right|+\left|\mathcal{R}_{2}\right|+\left|\mathcal{L}_{1}\right| \leq r+\ell
$$

and

$$
\left|\mathcal{R}_{1}\right|+\left|\mathcal{L}_{1}\right|+\left|\mathcal{L}_{2}\right| \leq r+\ell
$$

always hold. If $\left|\mathcal{R}_{2}\right| \geq\left|\mathcal{L}_{2}\right|$, then by (6) and the above property of $\mathcal{S}^{\prime}$, we have

$$
\begin{aligned}
\left|\mathcal{S}^{\prime}\right| & \leq n\left(\left|\mathcal{R}_{1}\right|+\left|\mathcal{L}_{1}\right|\right)+\ell\left|\mathcal{R}_{2}\right|+r\left|\mathcal{L}_{2}\right| \\
& =n\left(\left|\mathcal{R}_{1}\right|+\left|\mathcal{R}_{2}\right|+\left|\mathcal{L}_{1}\right|\right)+(\ell-n)\left|\mathcal{R}_{2}\right|+r\left|\mathcal{L}_{2}\right| \\
& \leq n\left(\left|\mathcal{R}_{1}\right|+\left|\mathcal{R}_{2}\right|+\left|\mathcal{L}_{1}\right|\right)+(\ell+r-n)\left|\mathcal{R}_{2}\right| \\
& <n(r+\ell),
\end{aligned}
$$

the strict inequality holds because $n>r+\ell$ and $\left|\mathcal{R}_{2}\right|+\left|\mathcal{L}_{2}\right|=\left|\mathcal{D}_{2}\right|>0$. Otherwise, $\left|\mathcal{R}_{2}\right|<\left|\mathcal{L}_{2}\right|$, by (7) and the above property of $\mathcal{S}^{\prime}$, we similarly obtain $\left|\mathcal{S}^{\prime}\right|<n(r+\ell)$. 
Thus, in both cases, we get $\left|\mathcal{S}^{\prime}\right|<n(r+\ell)$, contradicting $\left|\mathcal{S}^{\prime}\right| \geq n(r+\ell)$. Therefore, $\mathcal{D}_{1}=\emptyset$. In this case,

$$
\left|\mathcal{S}^{\prime}\right|=\sum_{A \in \mathcal{R}_{2}}\left|\mathcal{S}_{A}^{\prime}\right|+\sum_{B \in \mathcal{L}_{2}}\left|\mathcal{S}_{B}^{\prime}\right| \leq\left|\mathcal{R}_{2}\right| \ell+\left|\mathcal{L}_{2}\right| r \leq n(\ell+r)
$$

Equality implies that $\mathcal{R}_{2}=\mathcal{R}, \mathcal{L}_{2}=\mathcal{L}$, and $\left|\mathcal{S}_{A}^{\prime}\right|=\ell$ and $\left|\mathcal{S}_{B}^{\prime}\right|=r$ for all $A \in \mathcal{R}$ and $B \in \mathcal{L}$. From the structure it is seen that for any $A_{1}, A_{2} \in \mathcal{R}, \mathcal{S}_{A_{1}}^{\prime}=\mathcal{S}_{A_{2}}^{\prime}$ whenever $A_{1} \cap A_{2}=\emptyset$. Then, that $\mathcal{R}_{2}=\mathcal{R}$ implies that $\mathcal{S}_{A_{1}}^{\prime}=\mathcal{S}_{A_{2}}^{\prime}$ for any $A_{1} \cap A_{2}=\emptyset$, so the identical $\mathcal{S}_{A}^{\prime}$ is a star, that is, $\mathcal{S}_{A}^{\prime}=\mathcal{L}^{\prime}[i]$ for some $i \in X_{2}$. Hence the connectivity of $H[\mathcal{R}]$ implies $\mathcal{S}_{A}=\mathcal{L}^{\prime}[i]$ for all $A \in \mathcal{R}$. Similarly, $\mathcal{S}_{B}^{\prime}=\mathcal{R}^{\prime}[i]$ for any $B \in \mathcal{L}$. That is, $\mathcal{S}^{\prime}=\mathcal{H}[i]$ for some $i \in X_{2}$. This completes the proof of the first step.

We now prove (ii). For every cyclic permutation $\sigma$ of $[n]$ and $A \subset[n]$, we say $\sigma$ contains $A$ if $A$ is an interval. Define $\mathcal{R}_{\sigma}=\left\{A \in B_{n, r}: \sigma\right.$ contains $\left.A\right\}$ and $\mathcal{L}_{\sigma}=\{A \in$ $B_{n, \ell}: \sigma$ contains $\left.A\right\}$. Similarly, we may define $\mathcal{R}_{\sigma}^{\prime}$ and $\mathcal{L}_{\sigma}^{\prime}$. Let $\Gamma_{1}$ and $\Gamma_{2}$ be the set of all cyclic permutations of $[n]$ and $[n+1,2 n]$, respectively. It is well know that $\Gamma_{1}$ is a conjugate class in the symmetric group $S_{n}$, i.e., $\Gamma_{1}=\left\{\sigma^{\tau}: \tau \in S_{n}\right\}$ for each selected $\sigma \in \Gamma_{1}$. Here, $\sigma^{\tau}=\tau \sigma \tau^{-1}$.

For $\sigma \in \Gamma_{1}$ and $\eta \in \Gamma_{2}$, let $\mathcal{H}_{\sigma, \eta}=\left(\mathcal{R}_{\sigma} \times \mathcal{L}_{\eta}^{\prime}\right) \cup\left(\mathcal{L}_{\sigma} \times \mathcal{R}_{\eta}^{\prime}\right)$. Clearly, $\mathcal{F}=\bigcup_{\sigma \in \Gamma_{1}, \eta \in \Gamma_{2}} \mathcal{H}_{\sigma, \eta}$. Write $\sigma_{0}=(1,2, \ldots, n)$ and $\eta_{0}=(n+1, n+2, \ldots, 2 n)$. Then $\mathcal{H}=\mathcal{H}_{\sigma_{0}, \eta_{0}}$. For each $\sigma \in \Gamma_{1}$ and $\eta \in \Gamma_{2}$, by Lemma 2.2 and step (i), $\mathcal{S} \cap \mathcal{H}_{\sigma, \eta}=\mathcal{H}_{\sigma, \eta}[x]$ for some $x \in[2 n]$, which is denoted by $x_{\sigma, \eta}$. That is,

$$
\begin{aligned}
& \mathcal{S} \cap \mathcal{H}_{\sigma, \eta}=\left(\mathcal{R}_{\sigma} \times \mathcal{L}_{\eta}^{\prime}\left[x_{\sigma, \eta}\right]\right) \cup\left(\mathcal{L}_{\sigma} \times \mathcal{R}_{\eta}^{\prime}\left[x_{\sigma, \eta}\right]\right) \text { if } x_{\sigma, \eta} \in[n+1, n] \text {, or } \\
& \mathcal{S} \cap \mathcal{H}_{\sigma, \eta}=\left(\mathcal{R}_{\sigma}\left[x_{\sigma, \eta}\right] \times \mathcal{L}_{\eta}^{\prime}\right) \cup\left(\mathcal{L}_{\sigma}\left[x_{\sigma, \eta}\right] \times \mathcal{R}_{\eta}^{\prime}\right) \text { if } x_{\sigma, \eta} \in[n] .
\end{aligned}
$$

Without loss of generality, we may assume $x_{\sigma_{0}, \eta_{0}}=n+1$. To complete the proof we need only prove that $x_{\sigma, \eta}=n+1$ for all $\sigma \in \Gamma_{1}$ and $\eta \in \Gamma_{2}$.

Define a relation $\sim$ on $\Gamma_{1}: \sigma \sim \tau$ if $\tau=\sigma^{(i, \sigma(i))}$ for some $i \in[n]$. Here, $(i, j)$ denotes the transposition in $S_{n}$, which interchanges $i$ and $j$, and fixes other elements of $[n]$. This relation is clearly symmetric. We now prove that $x_{\tau, \eta}=x_{\sigma, \eta}$ if $\tau \sim \sigma$. By symmetry we may assume $\eta=\eta_{0}, \sigma=\sigma_{0}$ and $\tau=\sigma^{(i, i+1)}$. Suppose $x_{\tau, \eta_{0}}=x \neq n+1$. Then, from $r<\ell \leq \frac{n}{2}$ we see that $\mathcal{L}_{\tau} \times \mathcal{R}^{\prime}[x]$ and $\mathcal{L} \times \mathcal{R}^{\prime}[n+1]$ are not cross-intersecting if $x \in[n+1,2 n]$; and $\mathcal{R}_{\tau}[x] \times \mathcal{L}^{\prime}$ and $\mathcal{R} \times \mathcal{L}^{\prime}[n+1]$ are not cross-intersecting if $x \in[n]$. So $\mathcal{S} \cap \mathcal{H}_{\tau, \eta}$ and $\mathcal{S} \cap \mathcal{H}_{\sigma, \eta}$ are not cross-intersecting, contradicting that $\mathcal{S}$ is intersecting. Similarly, $x_{\sigma, \eta}=x_{\sigma, \gamma}$ if $\eta \sim \gamma$.

For $\sigma \in \Gamma_{1}$, it is easy to see that there exists a subset $\left\{\sigma_{1}, \sigma_{2}, \ldots, \sigma_{k}\right\}$ of $\Gamma_{1}$ such that $\sigma_{0} \sim \sigma_{1}, \sigma_{1} \sim \sigma_{2}, \ldots, \sigma_{k} \sim \sigma$. Similarly, for $\eta \in \Gamma_{2}$, there exists a subset $\left\{\eta_{1}, \eta_{2}, \ldots, \eta_{t}\right\}$ of $\Gamma_{2}$ such that $\eta_{0} \sim \eta_{1}, \eta_{1} \sim \eta_{2}, \ldots, \eta_{t} \sim \eta$. So we have $n+1=x_{\sigma_{0}, \eta_{0}}=\cdots=x_{\sigma_{k}, \eta_{0}}=$ $x_{\sigma, \eta_{0}}=\cdots=x_{\sigma, \eta}$, as required.

Acknowledgements The authors are greatly indebted to the anonymous referees for giving useful comments and suggestions that have considerably improved the manuscript. The first author is supported by the National Natural Science Foundation of China 
(No.11171224), Ph.D. Programs Foundation of Ministry of Education of China (No. 20093127110001) and Innovation Program of Shanghai Municipal Education Commission

(No.09zz134); the second author is supported by the National Natural Science Foundation of China (No.11001249).

\section{References}

[1] M.O. Albertson and K.L. Collins, Homomorphisms of 3-chromatic graphs, Discrete Math., 54 (1985) 127-132.

[2] R. Ahlswede, H. Aydinian and L.H. Khachatrian, The Intersection Theorem for Direct Products, European J. Combin., 19 (1998) 649-661.

[3] P.J. Cameron and C.Y. Ku, Intersecting families of permutations, European J. Combin., 24 (2003) 881-890.

[4] P. Erdős, C. Ko and R. Rado, Intersection theorems for systems of finite sets, Quart. J. Math. Oxford Ser., 2 (12) (1961) 313-318.

[5] P. Frankl, An Erdős-Ko-Rado Theorem for direct products, European J. Combin., 17 (1996) 727-730.

[6] X.B. Geng, J. Wang and H.J. Zhang, Structures of Independent Sets in Direct Products of Some Vertex-transitive Graphs, Acta Math. Sin. (Engl. Ser.), DOI: 10.1007/s10114-011-0311-5.

[7] G. O. H. Katona, A simple proof of the Erdős-Ko-Rado theorem, J. Combin. Theory Ser. B, 13 (1972) 183-184.

[8] C.Y. Ku and T.W.H. Wong, Intersecting families in the alternating group and direct product of symmetric groups, Electron. J. Combin., 14 (2007).

[9] C.Y. $\mathrm{Ku}$ and B.B. Mcmillan, Independent sets of maximal size in tensor powers of vertex-transitive graphs, J. Graph Theory, 60 (2009) 295-301.

[10] P.K. Jha and S. Klavžar, Independence in direct-product graphs, Ars Combin., 50 (1998) 53-60.

[11] B. Larose and C. Tardif, Projectivity and independent sets in powers of graph, J. Graph Theory, 40 (2002) 162-171.

[12] Mario Valencia-Pabon and Juan Vera, Independence and coloring properties of direct products of some vertex-transitive graphs, Discrete Math., 306 (2006) 2275-2281.

[13] C. Tardif, Graph products and the chromatic difference sequence of vertex-transitive graphs, Discrete Math., 185 (1998) 193-200.

[14] J. Wang and H.J. Zhang, Cross-intersecting families and primitivity of symmetric systems, J. Combin. Theory Ser. A, 118 (2011) 455-462.

[15] H.J. Zhang, Primitivity and independent sets in direct products of vertex-transitive graphs, J. Graph Theory, 67 (2011) 218-225.

[16] H.J. Zhang, Independent sets in direct products of vertex-transitive graphs, preprint, arXiv:1007.0797. http://arxiv.org/abs/1007.0797. 